\title{
Rheology of Polystyrene Solutions with Scarcely Entangled Chains; Role of Slow Relaxation Mode in Nonlinear Behavior
}

\author{
Tadashi INOUE, and Kunihiro OSAKI \\ Institute for Chemical Research, Kyoto University, Uji, Kyoto 611-0011, Japan \\ (Received : May 30, 2003)
}

\begin{abstract}
Linear viscoelasticity behavior was described with sum of two terms for semi-dilute polystyrene solutions in tricresyl phosphate with molecular weights $(M)$ close to $2 M_{\mathrm{e}}$, where $M_{\mathrm{e}}$ is the entanglement molecular weight in the solution. One was a Rouse-Zimm (RZ) term represented by the Rouse-Zimm theory with arbitrarily chosen value of the longest relaxation time $\left(\tau_{\mathrm{RZ}}\right)$. The other $\left(\mathrm{L}\right.$ term) consisted of a relaxation mode with single relaxation time, $\tau_{\mathrm{L}}\left(>\tau_{\mathrm{RZ}}\right)$, and high-frequency limiting modulus $\left(G_{\mathrm{L}}\right)$ proportional to square of concentration, $c$. The shear stress, $\sigma$, and the first normal stress difference, $N_{1}$, in steady shear were described with simple formulas proposed previously for more dilute solutions. The stress due to L term was assumed to be given by a K-BKZ equation with damping function $h(\gamma)=$ $\left(1+0.2 \gamma^{2}\right)^{-1 / 2}$, where $\gamma$ is magnitude of shear. Contributions to $\sigma$ and $N_{1}$ from RZ term were derived from the Rouse-Zimm model theory, in which the relaxation time in steady flow decreased with rate of shear, $\dot{\gamma}$, as $\tau_{\mathrm{st}}=\tau_{\mathrm{RZ}}{ }^{0}+\left(\tau_{\mathrm{RZ}}-\tau_{\mathrm{RZ}}{ }^{0}\right) /$ $\left(1+0.35 \tau_{\mathrm{RZ}} \dot{\gamma}\right)$. Here $\tau_{\mathrm{RZ}}{ }^{0}$ is the longest relaxation time of the polymer at the infinite dilution limit. The result together with previous result for more dilute solutions implies that $\eta$ and $\Psi_{1}$ for any non-entangled solutions are well described with use of parameters derived from dynamic viscoelasticity.
\end{abstract}

Key Words: Dilute polymer solution / Viscosity / Normal stress difference / Rouse model

\section{INTRODUCTION}

The purpose of the present study is to measure complex modulus and stresses in steady shear flow of polymer solutions with scarcely entangled chains and to examine whether or not simple phenomenological relations proposed for more dilute solutions ${ }^{1,2)}$ can be applied for non-entangled polymer solutions of various concentrations.

Linear viscoelasticity of very dilute polymer solutions is well described with spring-bead model theories. ${ }^{3,4)}$ Behavior of $\Theta$ solvent systems is described by the Zimm theory ${ }^{4,5)}$ for a Gaussian chain and with strong hydrodynamic interaction between segments (non-free draining Gaussian model). Behavior of good solvent systems shows some similarity to that derived from the Rouse theory, ${ }^{3,5)}$ based on the assumptions that polymer chain is a Gaussian chain and hydrodynamic interaction is not included (free draining Gaussian model). The apparent free draining behavior of good solvent systems is actually caused by excluded volume interaction between segments or non-Gaussian nature of chain. ${ }^{\text {) }}$

At finite concentrations, polymer chains overlap with one another. The degree of overlapping is represented with a reduced concentration, $c / c^{*}$, where the overlapping concentration, $c^{*}$, may be given by

$$
c^{*}=\frac{2.5}{[\eta]}
$$

Here [ $\eta]$ is intrinsic viscosity. Theories tell that the hydrodynamic interaction as well as the excluded volume interaction is shielded by chain overlapping and the viscoelastic behavior at finite concentrations resembles that of the Rouse theory. ${ }^{5,6)}$ Data of complex modulus have been described with the Rouse-Zimm theory regarding the hydrodynamic interaction parameter and the longest relaxation time as adjustable parameters. ${ }^{5}$

A recent detailed study revealed that the procedure was not quite satisfactory for polystyrene solutions in a good solvent, tricresyl phosphate, with $c / c^{*}=0.25-1.4$. Observed results could be fitted with

$$
\begin{aligned}
& G^{*}(\omega)=\frac{G_{L} \mathrm{i} \omega \tau_{L}}{1+\mathrm{i} \omega \tau_{L}}+\frac{c R T}{M} \sum_{p=1}^{N} \frac{\mathrm{i} \omega \tau_{p}}{1+\mathrm{i} \omega \tau_{p}}+\mathrm{i} \omega \eta_{\infty} \\
& \tau_{p}=\tau_{R Z} p^{-a}
\end{aligned}
$$

The last two terms of Eq.(2), which we name RZ term and $\eta_{\infty}$ term, respectively, correspond to those used in preceeding studies; ${ }^{5)} a=2$ in Eq.(3) for the Rouse theory and $a=1.5$ for the Zimm theory. The first term on the rhs, L term, was necessary to express experimental values. For a series of 
solutions with $c / c^{*}=0.25-1.4\left(M / \mathrm{kg} \mathrm{mol}^{-1}: 2890\right.$ and 8420$)$, experimental data of complex modulus were approximated by the equation with appropriate choice of fitting parameters, $\tau_{L}$, $G_{\mathrm{L}}, \tau_{\mathrm{RZ}}, a$, and $\eta_{\infty} . \tau_{\mathrm{RZ}}$ increased with $M$ and $c ; a$ varied from 1.7 to 2.0 with increasing $c ; \eta_{\infty}$ was equal to solvent viscosity. $\tau_{\mathrm{L}}$ was approximately equal to $5 \tau_{\mathrm{RZ}} ; G_{\mathrm{L}}$ was approximately equal to $0.22\left(c / c^{*}\right)(c R T / M)$. Addition of one $\mathrm{L}$ term was sufficient to reproduce the data. Reliability of the method was discussed in details in the previous paper. ${ }^{1)}$

Based on the new expression of complex modulus, a phenomenological model was proposed to derive the viscosity, $\eta(\dot{\gamma})$, and the first normal stress coefficient, $\Psi_{1}(\dot{\gamma})$, in steady shear flow from the parameters given above. ${ }^{2)}$ The stress due to $\mathrm{L}$ term was assumed to be given by a K-BKZ equation with damping function $h(\gamma)=\left(1+0.2 \gamma^{2}\right)^{-1 / 2}$, where $\gamma$ is magnitude of shear. Contributions to $\eta$ and $\Psi_{1}$ from RZ term were derived from the Rouse-Zimm model, in which the relaxation time in steady flow was assumed to decrease with the rate of shear, $\dot{\gamma}$, as $\tau_{\mathrm{st}}=\tau_{\mathrm{RZ}}{ }^{0}+\left(\tau_{\mathrm{RZ}}-\tau_{\mathrm{RZ}}{ }^{0}\right) /\left(1+0.35 \tau_{\mathrm{RZ}} \dot{\gamma}\right)$. Here $\tau_{\mathrm{RZ}}{ }^{0}$ is the longest relaxation time of the polymer at the infinite dilution limit. $\eta$ and $\Psi_{1}$ were well described with use of parameters derived from dynamic viscoelasticity.

Features of complex modulus and stresses in steady shear flow vary continuously with increasing concentration until the effect of entanglement becomes the dominant factor in rheological behavior. ${ }^{5)}$ The effect of entanglement is believed to be dominant if the molecular weight, $M$, is higher than $2 M_{\mathrm{e}}$, where $M_{\mathrm{e}}$ is the entanglement molecular weight in the solution. The solutions of previous studies were in the range $M$ $<M_{\mathrm{e}}$. Now we investigate the solutions with $M$ close to $2 M_{\mathrm{e}}$. This study, together with the earlier studies, covers whole range of concentration for polystyrene solution in tricresyl phosphate without entanglement effect.

\section{EXPERIMANTAL}

\subsection{Materials}

Test solutions are described in Table I. Polystyrene samples, f850 and $\mathrm{f} 288$, f128, and $\mathrm{f} 40$ with sharp molecular weight distributions were purchased from Tosoh Co., Ltd. The solvent, tricresyl phosphate, was purchased from Wako Co. and used as supplied. Preparation of solutions was described before. ${ }^{7,9)}$

Concentration and molecular weights of test solutions are shown with filled marks in Fig.1. Entanglement molecular weight was determined from the following equations. ${ }^{7)}$

$$
\left(c / 1000 \mathrm{~kg} \mathrm{~m}^{-3}\right)^{1.4} M_{\mathrm{e}}(c)=7.3 \mathrm{~kg} \mathrm{~mol}^{-1} \quad\left(\mathrm{c} \leq 100 \mathrm{~kg} \mathrm{~m}^{-3}\right)
$$

Table I Test solutions and viscoelastic parameters at $273 \mathrm{~K}$.

\begin{tabular}{|c|c|c|c|c|c|c|c|c|}
\hline code $^{\mathrm{a}}$ & $c / c^{*}$ & $M / M_{\mathrm{e}}$ & $\tau_{R Z} / \mathrm{s}$ & $A$ & $\tau_{L T} / \mathrm{s}$ & $G_{L T} / \mathrm{Pa}$ & $\eta_{\alpha} / \mathrm{Pas}$ & $\tau_{\mathrm{RZ}}{ }^{0} / \mathrm{s}^{\mathrm{b}}$ \\
\hline $\mathrm{f} 850-01$ & 2.79 & 2.1 & 2.0 & 2.0 & 6.5 & 1.7 & 0.47 & 0.55 \\
\hline $\mathrm{f} 288-02$ & 2.58 & 1.9 & 0.36 & 2.0 & 1.95 & 8.2 & 0.5 & 0.088 \\
\hline $\mathrm{f} 128-04$ & 2.56 & 1.9 & 0.090 & 2.0 & 0.43 & 72 & 0.7 & 0.016 \\
\hline $\mathrm{f} 40-10$ & 3.26 & 2.6 & 0.065 & 2.0 & 0.24 & 530 & 1.2 & 0.0033 \\
\hline
\end{tabular}

a. In sample codes, $\mathrm{f} 850, \mathrm{f} 288, \mathrm{f} 128$, and $\mathrm{f} 40$ represent polystyrene with molecular weights $8420,2890,1090$, and $427 \mathrm{~kg} \mathrm{~mol}^{-1}$, respectively. Figures following hyphen represent concentration in $\mathrm{g} \mathrm{cm}^{-3}$, as; 01: $0.01 \mathrm{~g} \mathrm{~cm}^{-3}$

b. $\tau_{\mathrm{RZ}}$ and $a$ values at infinite dilution limit were determined from intrinsic viscosity-molecular weight relation; $a^{0}=1.7$ at any molecular weight. ${ }^{1)}$

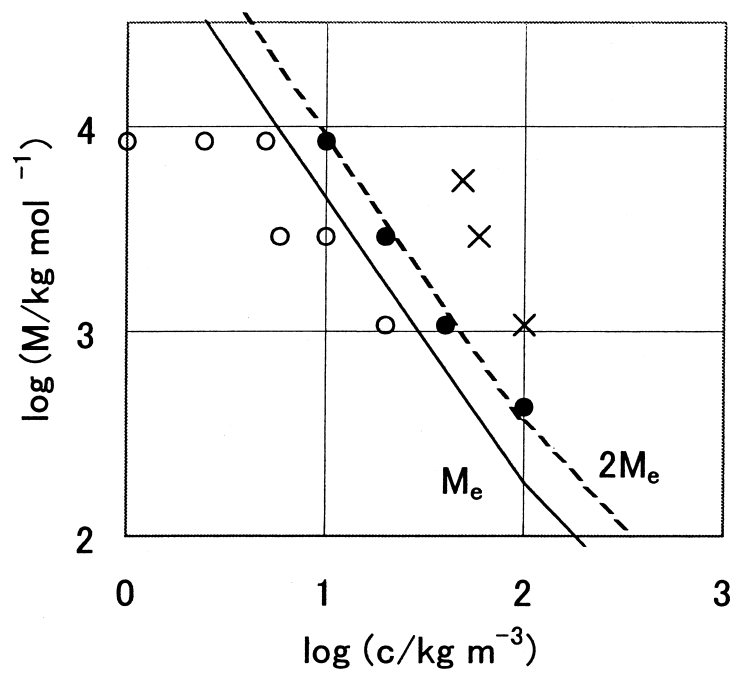

Fig.1 Concentration and molecular weight of test solutions (filled marks). Unfilled circles represent systems in previous studies with $c / c^{*}<1.5{ }^{1,2)}$ Crosses represent systems with typical entanglement effect. ${ }^{7,8)}$ Two lines show relations $M=M_{\mathrm{e}}$ and $M=$ $2 M_{\mathrm{e}}$, respectively, as determined with Eqs.(4) and (5).

$$
\left(c / 1000 \mathrm{~kg} \mathrm{~m}^{-3}\right) M_{\mathrm{e}}(c)=18 \mathrm{~kg} \mathrm{~mol}^{-1} \quad\left(\mathrm{c}>100 \mathrm{~g} \mathrm{~cm}^{-3}\right)
$$

Filled marks representing the test solutions lie close to the line for $M=2 M_{\mathrm{e}}$. Reduced concentrations, $c / c^{*}$, are shown in Table I; $c^{*}$ was determined from Eq.(1) with ${ }^{9)}$

$$
[\eta] / \mathrm{g} \mathrm{cm}^{-3}=7.2 \times 10^{-3}\left(\mathrm{M} / \mathrm{g} \mathrm{mol}^{-1}\right)^{0.72}
$$

Using Eq.(4), we obtain a convenient expression for polystyrene semidilute solutions in tricresyl phosphate

$$
\frac{M}{M_{\mathrm{e}}}=\frac{1}{2}\left(\frac{c}{c^{*}}\right)^{1.4} \quad\left(c \leq 100 \mathrm{~kg} \mathrm{~m}^{-3}\right)
$$


In the range of present study, the relation $M=2 M_{\mathrm{e}}$ (dashed line) corresponds to $c / c^{*}=2.7$.

Unfilled circles in Fig. 1 represent solutions with lower concentrations studied previously. The $\mathrm{x}$ marks represent some of the systems with typical behavior of entangled polymers. ${ }^{7-9)}$

\subsection{Measurements}

A standard rheometer (ARES; Rheometrics Scientific Far East) was used for all the measurements. Parallel plate fixture with $25 \mathrm{~mm}$ diameter was used for measurements of complex modulus. Measurements were performed at several temperatures in the range from $-30^{\circ} \mathrm{C}$ to $30^{\circ} \mathrm{C}$ and the data were reduced to a reference temperature, $0^{\circ} \mathrm{C}$, with the method of reduced variables. ${ }^{10)}$ Details of procedure and properties of shift factor have been reported. $1,7,8$ )

Cone-and-plate fixture with $25 \mathrm{~mm}$ diameter and 0.1 radian cone angle was used for measurements of shear stress, $\sigma$, and the first normal stress difference, $N_{1}$, in steady shear flow. Data obtained at temperatures other than $0^{\circ} \mathrm{C}$ were reduced to the reference temperature, $0^{\circ} \mathrm{C}$, with the method of reduced variables. Shift factors obtained for complex modulus were used. Details of procedure have been reported. ${ }^{2,8,9)}$

Shear stress after application of shear strain of magnitude $\gamma$ was measured for $\mathrm{f} 128-04$ at $-20^{\circ} \mathrm{C}$. Cone-and-plate fixture with $25 \mathrm{~mm}$ diameter and 0.1 radian cone angle was used. Strain-dependent relaxation modulus, $G(t, \gamma)$, was obtained as the ratio of stress to strain.

\section{EXPERIMENTAL RESULTS}

\subsection{Complex Modulus and Parameters for Linear Viscoelasticity}

Complex modulus for sample f128-04 is shown in Fig.2. Typical low frequency behavior for viscoelastic fluids, $G^{\prime} \propto \omega^{2}$ and $G^{\prime \prime} \propto \omega$, and typical high frequency behavior for nonentangled polymer solutions, $G^{\prime} \propto \omega^{a}$ and $G^{\prime \prime} \propto \omega$, are observed. The rubbery plateau zone, characterized by relatively slow increase of $\log G^{\prime}$ over a intermediate range of $\omega$, does not exist. Over a certain range around $\omega=30 \mathrm{~s}^{-1}, G^{\prime}$ is slightly higher than $G^{\prime \prime}$. As a whole, the complex modulus is characteristic for a system just at the onset of entanglement. ${ }^{\text {) }}$ This is consistent with the number of entanglement, 1.9, estimated for this system.

The fitting curves calculated from Eqs.(1) and (2) with the parameters shown in Table I are completely covered with data points. Procedure of fitting is the following. Gross values of $a$ and $\eta_{\infty}$ is determined from data at high $\omega$; contributions from

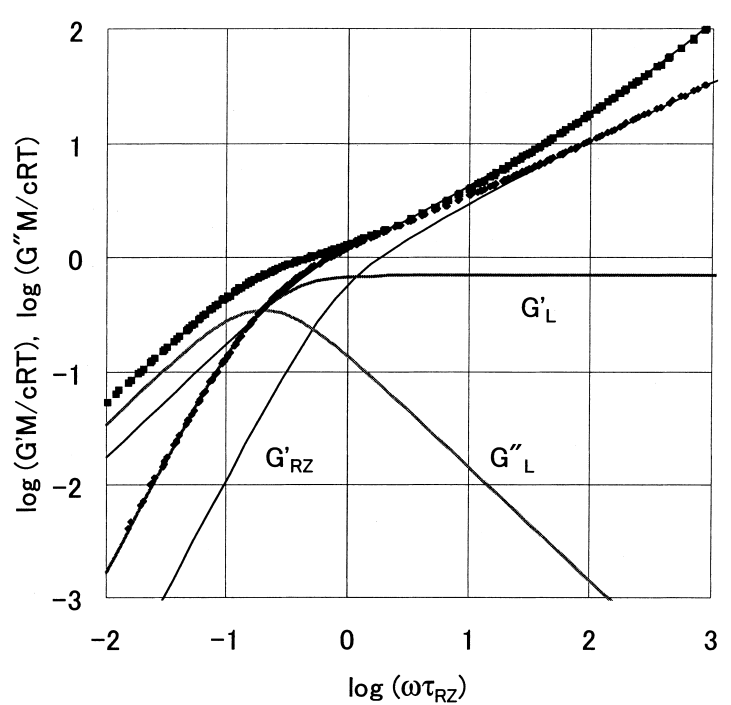

Fig.2 Complex modulus for f128-04 (dots). Lines for fitting function are completely covered with data points. Thin lines represent contributions from RZ term plus $\eta_{\infty}$ term and gray lines from $\mathrm{L}$ term.

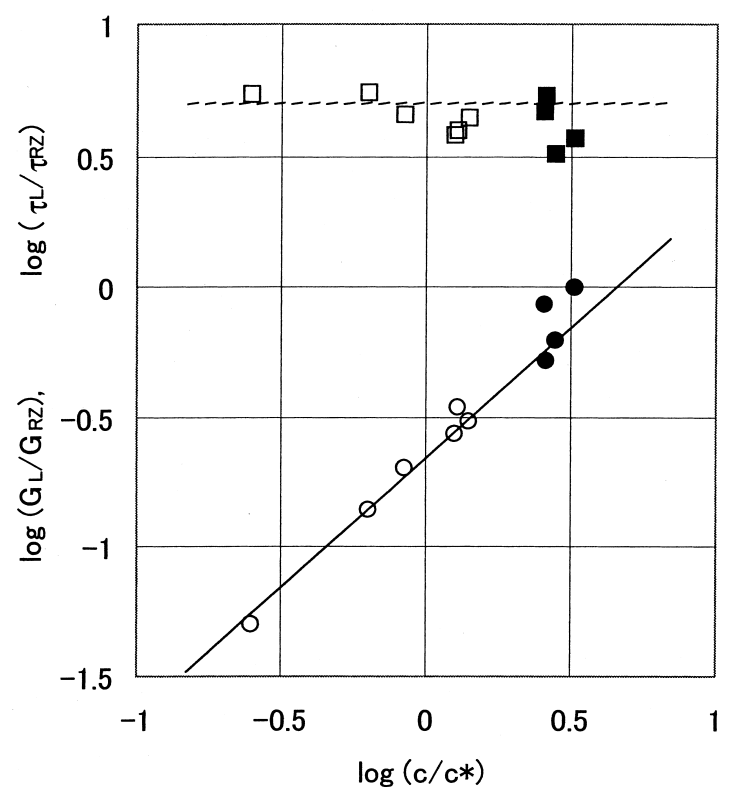

Fig.3 Ratios $G_{\mathrm{L}} / G_{\mathrm{RZ}}$ (circles) and $\tau_{\mathrm{L}} / \tau_{\mathrm{RZ}}$ (squares) as functions of reduced concentration $c / c^{*}$. Filled marks represent systems studied in this paper and unfilled marks, those of previous studies. Solid line: $G_{\mathrm{L}} / G_{\mathrm{RZ}}=0.22 \mathrm{c} / \mathrm{c}^{*}$; dashed line: $\tau_{\mathrm{L}} / \tau_{\mathrm{RZ}}=4.6$.

RZ term and $\eta_{\infty}$ term is then calculated with a tentative value of $\tau_{\mathrm{RZ}}$; then we add $\mathrm{L}$ term with tentative values of $G_{\mathrm{L}}$ and $\tau_{\mathrm{L}}$. Parameters are varied until good fit is attained over whole range of $\omega$. Thin lines represent contributions from $\mathrm{RZ}$ term and $\eta_{\infty}$ term. Deviation from data points is quite large at low frequencies but addition of $\mathrm{L}$ term with only one relaxation time is sufficient to remove it.

Shapes of curves for complex modulus for other samples in 

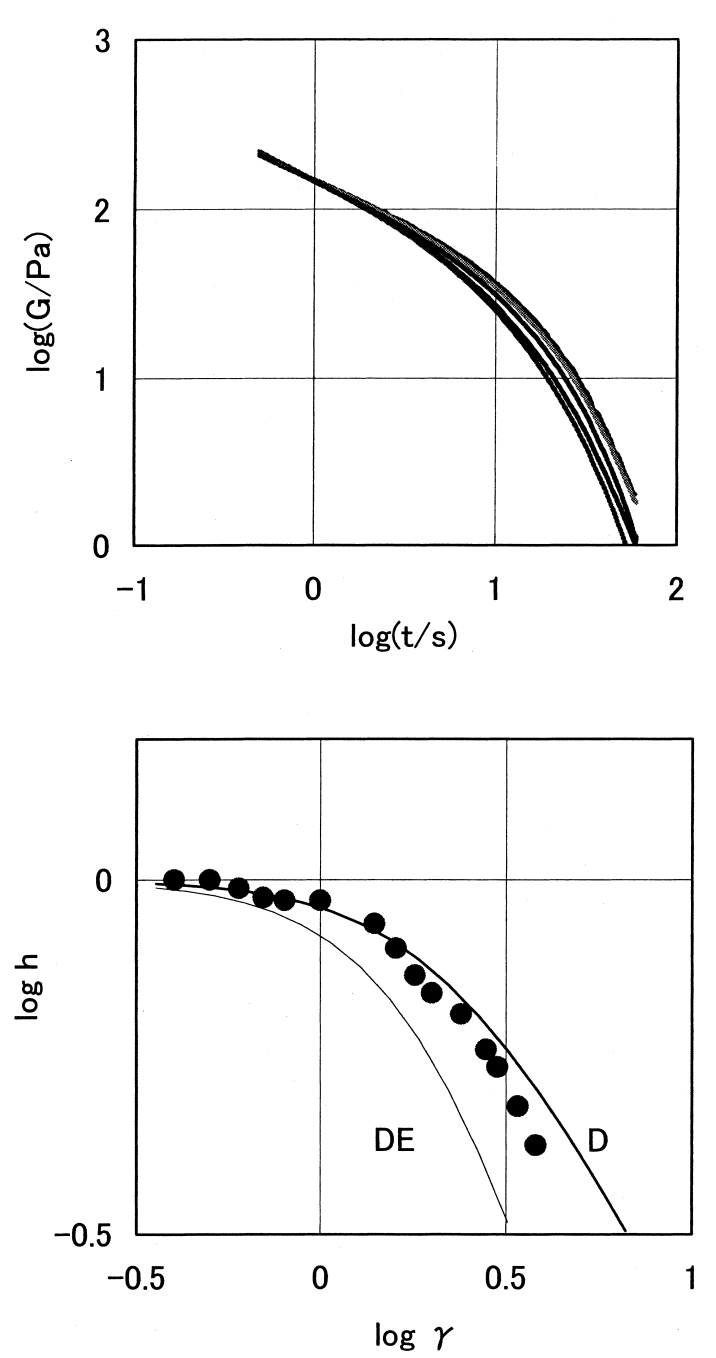

Fig.4 Relaxation modulus for f128- 04 at $-20^{\circ} \mathrm{C} ; \gamma=0.4,1.0,2.0,3.0$, and 4.0 from top (upper panel) and damping function; line $\mathrm{D}$ from fitting function (Eq.(15)) for dilute solutions and line DE from Doi-Edwards theory (lower panel).

Table I are similar to those in Fig.2. Scales of abscissa and ordinate are different from sample to sample. The difference may be easily seen in Table I, where fitting parameters are shown for all the samples. For systems with approximately the same $M / M_{\mathrm{e}}$, scale of time, represented by $\tau_{\mathrm{RZ}}$ and $\tau_{\mathrm{L}}$, is large for high $M$ and low $c$; scale of modulus, represented by $G_{\mathrm{RZ}}$ (= $c R T / M)$ and $G_{\mathrm{L}}$, is large for low $M$ and high $c$.

The parameters are compared with those for more dilute solutions of the previous study. ${ }^{1)}$ In Fig. 3 the ratios $\tau_{\mathrm{L}} / \tau_{\mathrm{RZ}}$ and $G_{\mathrm{L}} / G_{\mathrm{RZ}}\left(=G_{\mathrm{L}} M / c R T\right)$ are plotted against reduced concentration $c / c^{*}$. Points for the present samples are well on the extension of lines for more dilute solutions. Lines correspond to

$$
\begin{aligned}
& \frac{\tau_{L}}{\tau_{R Z}}=4.6 \\
& \frac{G_{L}}{G_{R Z}}=0.22 \frac{c}{c^{*}}
\end{aligned}
$$
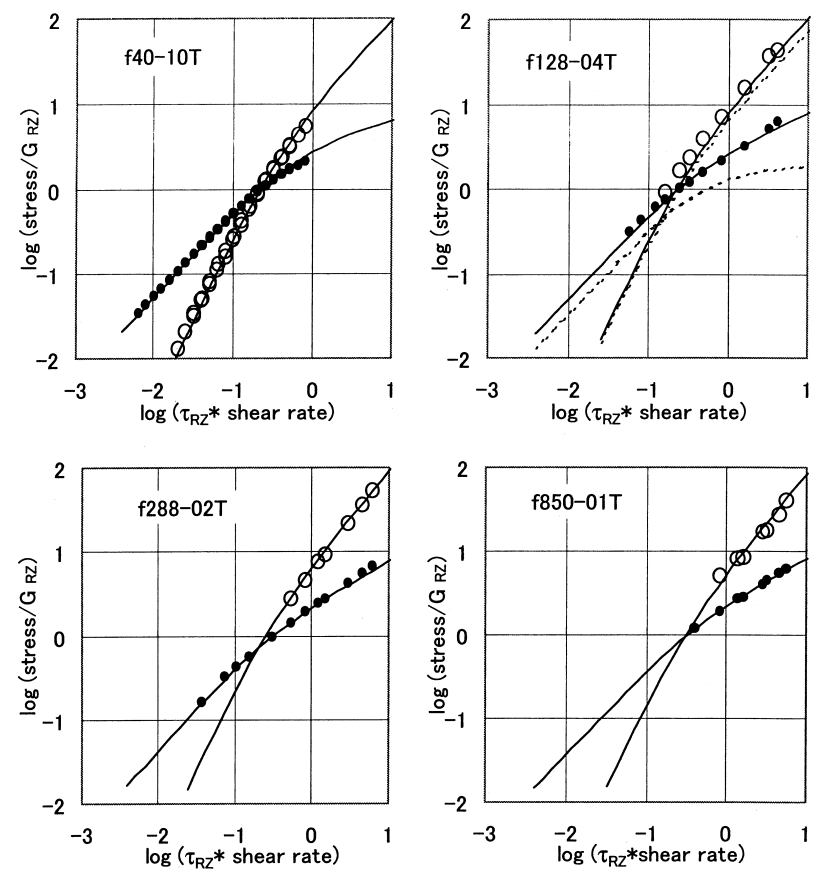

Fig.5 Shear stress (filled circles) and normal stress difference (unfilled circles) in steady shear flow. Solid lines represent fitting functions. Dashed lines for f128-04 represent contributions from L term.

One difference from more dilute solutions is that $a$ value is always 2 for systems with $M \approx 2 M_{\mathrm{e}}$. This is consistent with earlier works. ${ }^{5,7)}$

\subsection{Strain-Dependent Relaxation Modulus}

Strain-dependent relaxation modulus, $G(t, \gamma)$, for f128-04 at $-20^{\circ} \mathrm{C}$ is shown in Fig.4. Result at low $\gamma$ is consistent with dynamic data of Fig.2 if reduced to the reference temperature, $0^{\circ} \mathrm{C}$, using the shift factor, $\log a_{T}=1.38 . G(t, \gamma)$ could be factorized as $G(t, \gamma)=G(t) h(\gamma)$ at long times, $\log (t / s)>0.3$. The damping function, $h(\gamma)$, is shown in the lower panel of the figure.

\subsection{Stresses in Steady Shear Flow}

Shear stress and the first normal stress difference in shear flow of constant rate of shear were described before for 1850 $01^{11)}$ and for samples with lower concentrations. ${ }^{12)}$ Qualitatively the same behavior was observed for all the samples: stress overshoot at high $\dot{\gamma}$ and regular (reproducible) flow instability at still higher $\dot{\gamma}$.

Results for steady shear, $\sigma(\dot{\gamma})$ (filled circles) and $N_{1}(\dot{\gamma})$ (unfilled circles), are shown in Fig.5. They exhibit typical features for semidilute polymer solutions: $\sigma(\dot{\gamma}) \propto \dot{\gamma}$ and $N_{1}(\dot{\gamma}) \propto$ $\dot{\gamma}^{2}$ at low $\dot{\gamma}$ and weaker variations at high $\dot{\gamma}$. $\sigma(\dot{\gamma})$ at high $\dot{\gamma}$ is much higher than expected from the Cox-Merz empirical rule. ${ }^{13)}$ 


\section{DISCUSSION}

\subsection{Comparison of Steady Shear Data with Proposed Formula}

The formula proposed in the previous paper ${ }^{2}$ is as follows:

$$
\begin{aligned}
& \sigma(\dot{\gamma})=\sigma_{L}(\dot{\gamma})+\sigma_{R Z}(\dot{\gamma})+\dot{m}_{\infty} \\
& N_{1}(\dot{\gamma})=N_{1 L}(\dot{\gamma})+N_{1 R Z}(\dot{\gamma})
\end{aligned}
$$

where the $\mathrm{L}$ terms are given by

$$
\begin{aligned}
& \sigma_{L}(\dot{\gamma})=\dot{\gamma} \int_{0}^{\infty} G_{L} h(\gamma) s \exp \left(-s / \tau_{L}\right) \mathrm{d} s \\
& N_{1 L}(\dot{\gamma})=\dot{\gamma}^{2} \int_{0}^{\infty} G_{L} h(\gamma) s^{2} \exp \left(-s / \tau_{L}\right) \mathrm{d} s \\
& \gamma=\dot{\gamma} s \\
& h(\gamma)=\frac{1}{\left(1+0.2 \gamma^{2}\right)^{1 / 2}}
\end{aligned}
$$

and the RZ terms by

$$
\begin{aligned}
& \sigma_{R Z}(\dot{\gamma})=\frac{c R T}{M} \tau_{s t} S(a) \dot{\gamma} \\
& N_{1 R Z}(\dot{\gamma})=\frac{2 c R T}{M} \tau_{s t}{ }^{2} S(2 a) \dot{\gamma}^{2} \\
& S(x)=\sum_{p=1}^{\infty} p^{-x} \\
& \tau_{s t}=\tau_{R Z}{ }^{0}+\frac{\tau_{R Z}-\tau_{R Z}{ }^{0}}{1+0.35 \tau_{R Z} \dot{\gamma}}
\end{aligned}
$$

Here $\tau_{\mathrm{RZ}}{ }^{0}$ is the $\tau_{\mathrm{RZ}}$ value at the limit of infinite dilution and the $a$ value in Eqs.(16) and (17) is the average of $a$ values at the relevant concentration and at the infinite dilution limit.

These equations and the data in Table I are sufficient to evaluate $\sigma(\dot{\gamma})$ and $N_{1}(\dot{\gamma})$ in steady shear flow. The results are shown with solid lines in Fig.5. Agreement with the experimental data is excellent. The contribution from the $\mathrm{L}$ term is shown with dashed lines for f128-04. Since the relative concentration, $c / c^{*}$, is approximately the same for all the solutions in Figure 5, relative values of stresses, $N_{1 \mathrm{~L}} / N_{1}$ and $\sigma_{\mathrm{L}} /$ $\sigma$, at a reduced shear rate, $\tau_{\mathrm{RZ}} \dot{\gamma}$, are approximately the same for all the panels. It is seen that $N_{1}$ is mostly determined by the $\mathrm{L}$ term but $\sigma$ involves a considerable contribution from the RZ term.

The result exhibits that the proposed expression for stresses in steady shear is applicable to wide range of concentrations of non-entangled polymer solutions.

\subsection{Behavior of Damping Function}

The damping function of Eq.(15) was obtained in search for the functional form of $\mathrm{L}$ term for steady shear for relatively dilute solutions, $c / c^{*}<1.4$. The relaxation modulus was not measurable at such concentrations. It was measurable for the present systems with relatively high $c R T / M$ values: $440-10 \mathrm{~T}$, f128-04T, and f288-02T. The results are similar to each other. The damping function shown in Fig.4 is quite close to the value from Eq.(15), the line marked D. Thus the assumption of the K-BKZ behavior for the L term may be reasonable for the case of $M$ close to $2 M_{\mathrm{e}}$.

It seems that the same damping function approximated by Eq.(15) is applicable to any non-entangled polymer solutions. It may also be noted that it is close to the square root of the one derived from the Doi-Edwards theory, ${ }^{14)}$ the line marked DE in Fig.5.

\section{CONCLUSION}

The proposed formula is the only one that describes the shear thinning completely with the data of linear viscoelasticity for non-entangled polymer solutions as far as the author is informed. We revealed here that the formula is applicable to whole $c-M$ range of non-entangled systems of polystyrene in tricresyl phosphate. The simple form of the model and the common damping function for wide $c-M$ range are hopefully the key to understanding of molecular dynamics and rheology of non-entangled systems.

\section{Acknowledgement}

This study is a small fruit from the orchard cultivated by the late Professor John D. Ferry. KO is grateful to him for the personal education in his laboratory.

\section{REFERENCES}

1) Osaki K, Inoue T, Uematsu T, J Polym Sci Polym Phys Ed, 39, 211 (2001).

2) Osaki K, Inoue T, Uematsu Y, Yamashita T, J Polym Sci Polym Phys Ed, 40, 1038 (2002).

3) Rouse PE, J Chem Phys, 21, 1272 (1953).

4) Zimm BH, J Chem Phys, 24, 269 (1956).

5) See for example, Ferry JD, "Viscoelastic Properties of Polymers", 3rd ed, (1980), Chap. 9, Wiley, NY.

6) See for example, Doi M, Edwards SF, "The Theory of Polymer Dynamics”, (1986) pp. 108-116, University Press, Oxford 
7) Osaki K, Inoue T, Uematsu Y, Yamashita T, J Polym Sci Polym Phys Ed, 39, 1704 (2001).

8) Inoue T, Yamashita Y, Osaki K, Macromolecules, 35, 1770 (2002).

9) Osaki K, Inoue T, Uematsu Y, J Polym Sci Polym Phys Ed, 38, 3271 (2000).

10) ref. 5, Chap. 11.
11) Osaki K, Inoue $\mathrm{T}$, Isomura $\mathrm{T}, J$ Polym Sci Polym Phys Ed, 38, 2043 (2000).

12) Osaki, K, Inoue T, Isomura T, J Polym Sci Polym Phys Ed, 38, 1917 (2000).

13) Cox WP, Merz EH, J Polym Sci Polym Phys Ed, 28, 619 (1958).

14) Doi M, Edwards SF, J Chem Soc Faraday Trans II, 74, 1802 (1978), ibid, 74,1818 (1978), ibid, 75, 32 (1979) 